\title{
Heart arrhythmia detection using labview GUI based approach
}

\author{
Abhijeet R. Satani ${ }^{*}$, Dinesh R. Damodar ${ }^{2}$ and Bhoomi R. Satani ${ }^{3}$ \\ Founder \& Director, Limbable Biotechnology Private Limited, Gujarat, India ${ }^{1}$ \\ Ex-Student, Gujarat Technological University, Gujarat, India ${ }^{2}$ \\ Director, Limbable Biotechnology Private Limited, Gujarat, India ${ }^{3}$
}

\section{(C)2018 ACCENTS}

\begin{abstract}
This paper impose a practical approach and experiment to detect heart abnormality by placing surface electrode on the body of patient user and exerting use of LabView GUI to estimate about heart condition. Every disease can be treated and cure from substantive, if it got noticed and expressed to corresponding physician in the initial period of its origin. In contemporary era, traditional ECG device with graph structure output is in use to identify arrhythmia which need to be prescribed by physician in case patient came up with any inquire regarding relevant symptom. Arrhythmia has been found to be having such large impact with aging, so at old age people are more likely to get affected in major cases. Even people attempt to ignore visit physician in case of tiny difficulties happen to them considering it might be hallucinations due to heavy load work or lack of sufficient rest. Objective of this technique is to design and develop a technique to determine heart abnormality more particularly arrhythmia at early stage. Since it controlled by visual and graphical approach, it become familiar due to easy accessibility and interpreting features. Electrocardiography has captured here using circuitry mechanism by configuring proper values of various implemented filter and noise offset values. It gives real time alert about heart arrhythmia which has considerably responsible and involved for sudden deaths of victim. Currently available ECG devices enough expensive and only can be used under observation of experts don't allow old age people to access this test at home. Considering this perspective less expensive model with privileges of customization is employed and expressed here for prompt and regular check-up at home safely. So even in case of initial traits, they can immediately contact nearest physician for further guidance. Arrhythmia proliferates immediately or suddenly so early detection of arrhythmia detection can assist heart experts for iterating advance precautions.
\end{abstract}

\section{Keywords}

Heart arrhythmia detection, Heart abnormality detection, LABVIEW based GUI interface configuration, Electrocardiography, ECG acquisition circuit, Real time ECG analysis.

\section{Introduction}

According to the centre for disease control, cardiac arrhythmia remains the number one cause of death in the United States, claiming the lives of approximately 610,000 individuals every year [1]. Heart is responsible for accumulating deoxygenated blood from entire body and circulating the oxygen rich blood to the entire cells to nourish them. Arrhythmia is abnormal condition of the heart revealing sudden inclined or declined towards efficiency and rhythm of sinoatrial node to consistently conduct electrical impulses are needed to pump the heart normally. Sometimes heartbeat increases due to goal oriented activity of the user, it seems to be normal since heart need to circulate more blood in entire body in order to keep that activity steady, because eventually oxygen in blood will be utilized.

\footnotetext{
*Author for correspondence
}

452
By the cells of the muscle will proliferate the need of purify more oxygen from blood in body within short time. This event seems to be frequent and normal in athletes and user who does activity which requires more blood purification in few time.

Simultaneously it can create trouble in excluded case since it revels inability of heart to contract and expand according to necessity of individual pertaining to cardiac arrest in major cases. Cardiac arrest in adult age due to cardiac arrest is increasing same as increase in age, so there are more chances of being victim accidently after certain ages [2]. Arrhythmia detection has achieved here by acquiring electrocardiography signals which is consequences of electrical activity of the heart. SA node located at the upper right atrium and recognize to be natural pacemaker of the heart, is the inception among entire procedure of generating electrical activity of the heart sequentially [3]. Numerous methods are available 
based on heart rate variability analyse heart rate on time domain, frequency domain and non-linear parameters to classifying heart rate [4]. Normal ECG and heart rate of healthy patient lies in the range of 70-90 beats per minute. Exceeding or lowering these limits consequently increases chances of arrhythmia. Complete ECG waves consist of various segment identified and symbolized on graph according to its point location which tends to higher and low as $\mathrm{P}, \mathrm{Q}, \mathrm{R}, \mathrm{S}, \mathrm{T}$. Where $\mathrm{P}$ represent depolarisation of the atrial, QRS refers to depolarisation of the ventricles and $\mathrm{T}$ present repolarisation of the ventricles [5]. Normal ECG takes nearly 0.8 seconds to complete single cycle of PQRST wave. To distinguish and analyse ECG signal it can be divided in to several segment like R-R interval, P-R interval and peak to peak interval. Abnormal ECG waves can be classified in certain categories of arrhythmia by measuring beats per minute of victim. It recognizes to be bradycardia if found to be slower and tachycardia if found to be faster than normal beats mentioned above as well fibrillation seems to be faster uncoordinated heart beats [5] . Normal values associated with hearts ECG waveform has mentioned below in the form of Table 1 [6]. Table 2 shows the normal time interval of ECG.

Table 1 Normal value of ECG amplitude

\begin{tabular}{ll}
\hline Amplitude & \\
\hline Wave & Amplitude \\
\hline $\mathrm{P}$ & $0.25 \mathrm{mV}$ \\
$\mathrm{R}$ & $1.60 \mathrm{mV}$ \\
$\mathrm{Q}$ & $25 \%$ of R wave \\
$\mathrm{T}$ & $0.1-0.5 \mathrm{mV}$ \\
\hline
\end{tabular}

Table 2 Normal time interval of ECG

\begin{tabular}{ll}
\hline Duration & \\
\hline P-R interval & $0.12-0.20 \mathrm{sec}$ \\
Q-T interval & $0.35-0.44 \mathrm{sec}$ \\
S-T segment & $0.05-0.15 \mathrm{sec}$ \\
P wave interval & $0.11 \mathrm{sec}$ \\
QRS interval & $0.09 \mathrm{sec}$ \\
\hline
\end{tabular}

To determine this abnormality we had developed ECG acquisition circuit by placing electrodes and configuring band pass filter to only acquire associated signal along with ECG and minimizes signal to noise ration. Implemented controller embedded with analogue to digital converter and serial data communication support to interface with LABVIEW. Intuitive Graphical user interface to control various parameters of input according to circumstances.

\section{Research methodology}

Arrhythmia detection using ECG along with LABVIEW approach incorporate several aspects along with sequence has mentioned below in the form of block-diagram. The main objective of this phenomenon is to recognize abnormality of the heart by discriminating ECG waveforms and interval of PQRST waveform during each cycle. This phenomenon executed by acquiring and extracting real time ECG signal and pass through LabView based algorithms to smoothen it and achieve result of condition of user or victim. Figure lshows the block diagram of the proposed methodology.

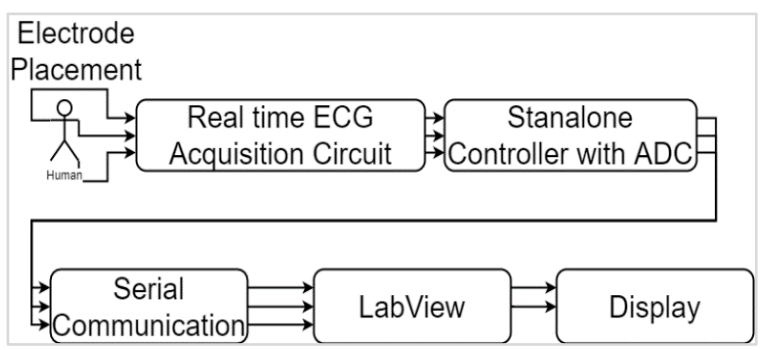

Figure 1 Block diagram of proposed methodology

Flowchart iterates the whole procedure since its inception of acquiring real time signal, smoothing signal using filters and eliminate noise from main line using notch filter as well summing amplifier because controller only distinguish between positive values. Figure 2 shows the electrode placement for ECG [1].

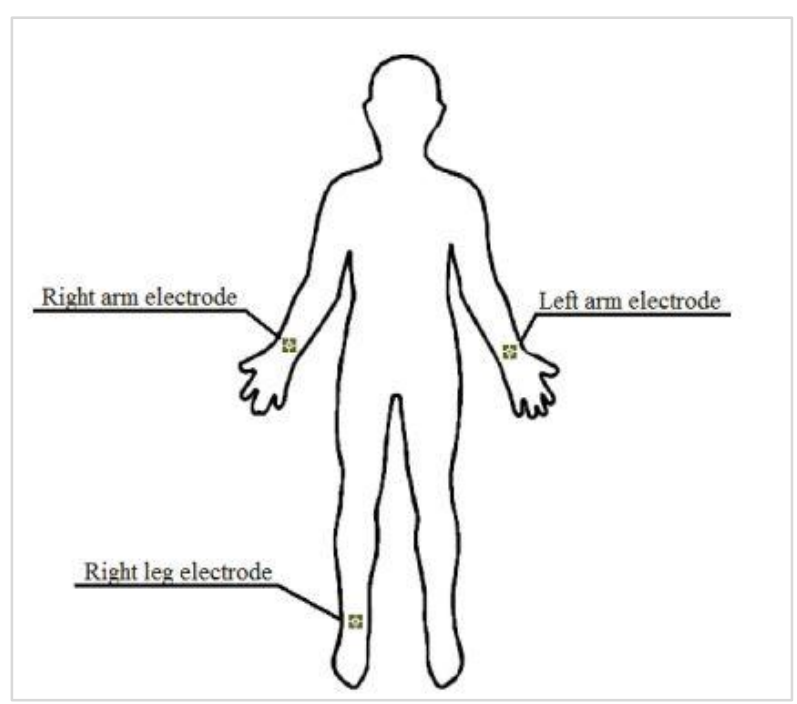

Figure 2 Electrode placement for ECG [1] 
Implemented placement of electrodes has depicted above in schematic [1] as more complex configuration of electrode placement can be done on various places according to Lead I, Lead II, Lead II, aVF, aVL, aVR configuration also recognize to be Einthoven's triangle. Both disposable and nondisposable electrodes can be used for this purpose. Controller along with inbuilt analogue to digital converter is easy to use and avoid external circuitry of ADC. Plug and play support of serial communication is recommended for this purpose so it doesn't required any external RS232 or USB protocol circuits to connect with LabView environment.

\subsection{Real-time ECG acquisition circuit}

Real time ECG acquisition is formed by depth technical detailing of existing ECG signal across various points of the body that acquired using surface electrode either disposable or non-disposable. Electrodes are attached according to Einthoven's configurations are connected with acquisition circuitry consist of high-gain Instrumentation amplifier, Universal filter and Notch filter. Main consideration behind designing of ECG circuit is designing of amplifier module which is able to boost the energy range of frequency lies in the dominantly in the range of $0 \mathrm{~Hz}$ to $500 \mathrm{~Hz}$ that can be enough distinguished by ADC of controller for sampling purpose.

2.1.1Pre-amplification

Figure 3 shows the circuit schematic of pre-amplifier based on INA128P.

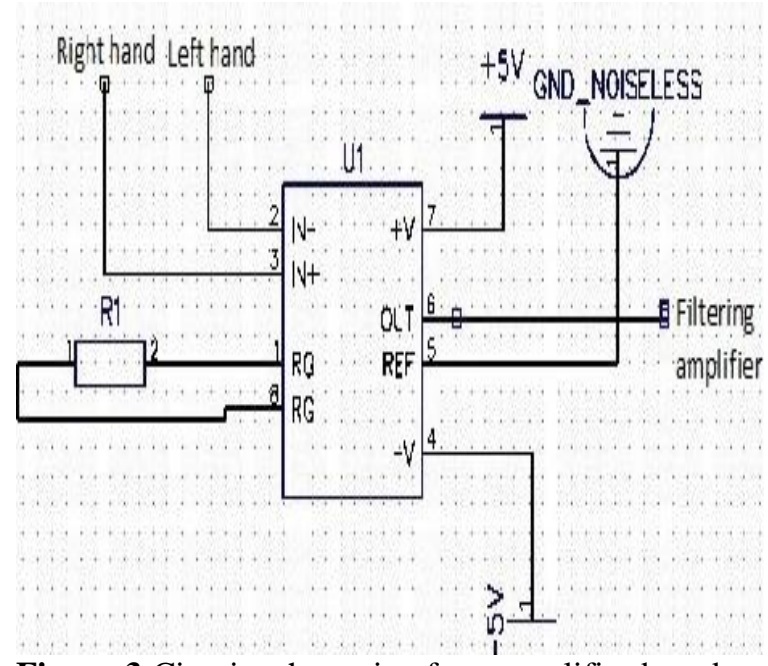

Figure 3 Circuit schematic of pre-amplifier based on INA128P
Pre-amplification procedure is required and recommended since electrical activity of the heart generated by the heart are of very low magnitude and in range of millivolts which is not falling in sensitivity criteria of the controller. So to bring out electrical pulses in adequate level which can be converted in to digital and easily distinguishable by controller to judge the rhythm of heart waves and forward it to filtering procedure. It is required to be having high Common mode rejection ratio and high sensitivity to even low frequency signal range. Dual in package IC INA128P has utilized here which fulfils the basic criteria for this purpose. The main considerations behind electing INA128P, chips lying in this series are general purpose chip containing low power instrumentation amplifier with great accuracy [7].

2.1.2Filtering

Noise is involved in signal acquisition by default because of such motion artefacts, inappropriate electrode placement, high, less contact between skin and electrode etc. Hence this acquired signal are of very low voltage and even such spikes of unpredicted frequency can demolish the chances of getting true results and depriving from ECG.

As described in figure band pass filter has employed using universal IC to compact size of the circuit. UAF42 is operational amplifier developed by Texas Instruments allows flexibility to configure entire band pass filter using the same pack of IC. To configure band pass filter with remarkable ECG frequency range of $0 \mathrm{~Hz}$ to $500 \mathrm{~Hz}$ and to smoothen signal using manually by settling values resistor, potentiometer has used, but it has set with $22 \mathrm{M} \Omega$ to get optimize result in the range of $0 \mathrm{~Hz}$ to $500 \mathrm{~Hz}$. Considered resistor calculation has mentioned below.

Band Pass Filter Range: $0 \mathrm{~Hz}-500 \mathrm{~Hz}$ $f_{H}$ : High Cut of frequency $f_{L}:$ Low Cut of Frequency

Center Frequency : $f_{c}=\sqrt{f_{H} f_{L}}$

Quality Factor : $Q=\frac{f_{c}}{f_{H}-f_{L}}$

$H_{0}$ : Center Frequency gain/Quality Factor

$$
R_{f 1}: \frac{1}{2 \pi c_{1} f_{0}}
$$

Figure 4 shows the schematic of passband filter. 


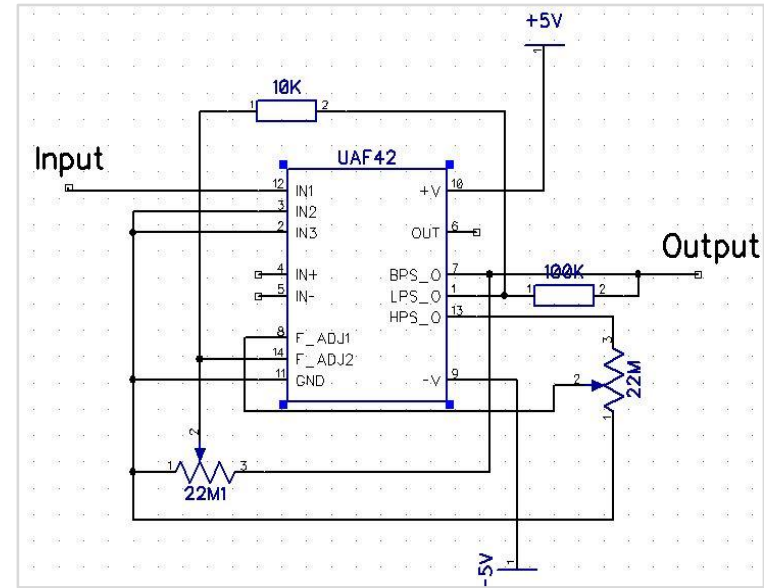

Figure 4 Schematic of passband filter

Utilizing this method and substituting value of required range of filter, we had found that about $22 \mathrm{M} \Omega$ resistor can be used to achieve passband of $0 \mathrm{~Hz}$ to $500 \mathrm{~Hz}$.

2.1.3Level shifting of signal

Figure 5 shows the schematic of level shifter.

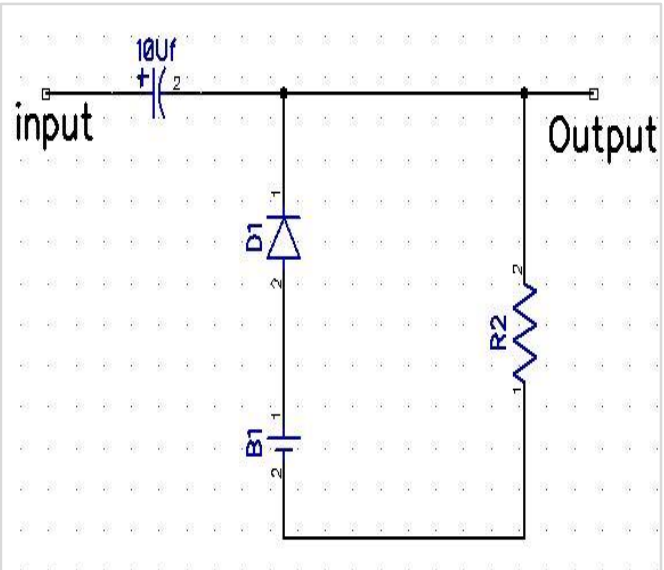

Figure 5 Schematic of level shifter

To distinguish ECG waveform in controller it is compulsory to shift all the negative values in the positive quarter otherwise controller will omit all the negative values since it lacks capability to work with negative waveform. So in order to get signal perceived and discriminate by controller level shifting has utilized here to shift level of waveform in positive domain.

\subsubsection{Serial communication}

Serial communication is the primary necessity to transfer signals from controller to the LabView environment which process it virtually and give significant results. Exempting inbuilt feature of serial communication it requires to install external module of serial like RS232 which increase complexity of entire circuit so Arduino Nano has employed here as controller comprises of atmega328p microchip giving fascinated with Serial chip and socket and allow access with plug and play feature.

2.1.5LabView environment

LabView environment utilises graphical user interface and graphical programming (flow programming) to achieve meaningful result of this experiment. It uses virtual instrument software architecture (VISA) drivers to receive ECG signal from controller in the form of serial data together with advanced signal processing toolkit (ASPT). Figure 6 shows the overview of ideal front panel designed and employed.

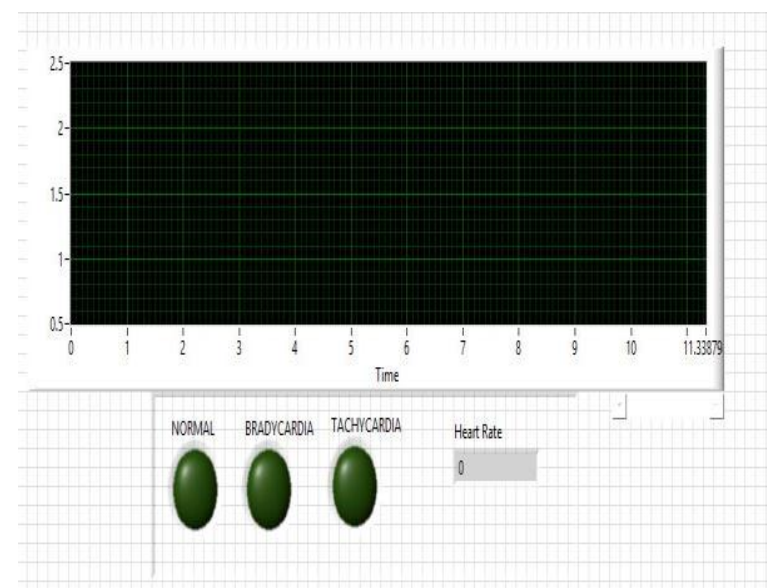

Figure 6 Overview of ideal front panel designed and employed

2.1.6Receive signal from controller

Figure 7 shows the ECG signal acquisition using VISA.

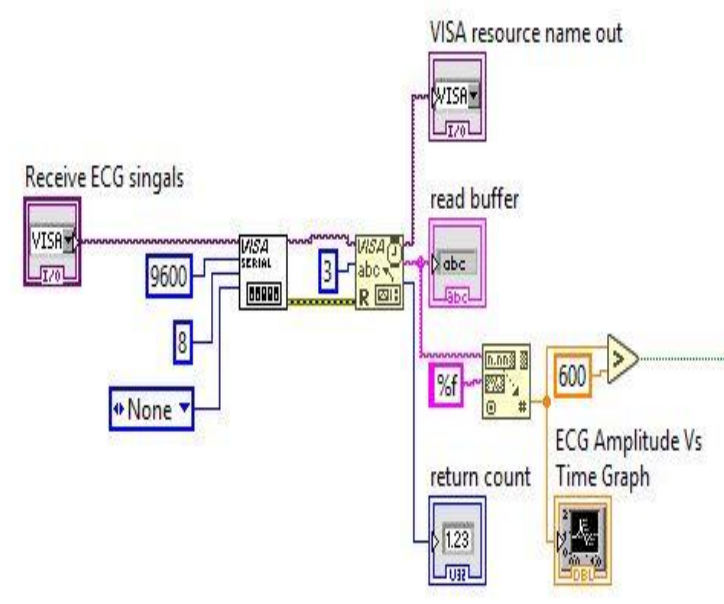

Figure 7 ECG signal acquisition using VISA 
Signal has received in LabView by using VISA drivers, Baud rate utilized for serial communication is 9600,8 bit data transmission with no encryption of odd or even parity which revels output on graph as ECG Amplitude VS Time Graph and simultaneously gives highest peak value as string output.

2.1.7Looping of pre-calculated minute

Figure 8 shows the accurate 1 minute loop designing for judgement purpose.

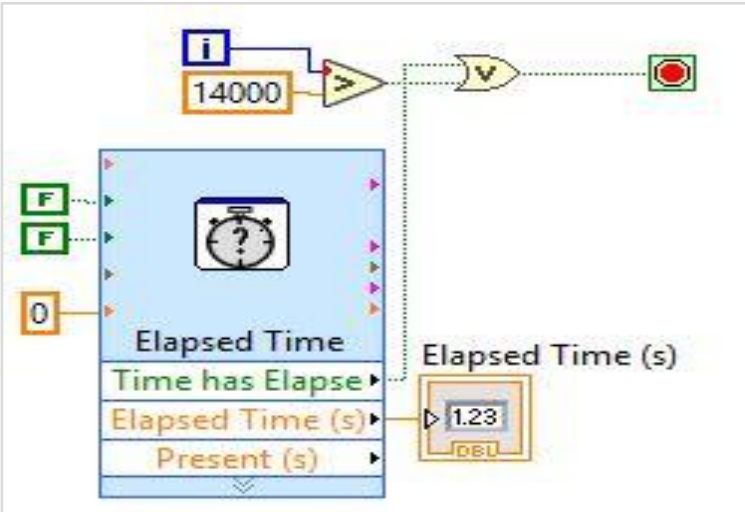

Figure 8 Accurate 1 minute loop designing for judgement purpose

In order to categorize various segment of the ECG waveform in time domain, it recommend use of RTC (Real time clock since timing of the controller has been judged by the internal timer of the controller so it might show remarkable or slight changes than real time). RTC gives access to the real time environment can give consideration to judge about heart rate variability according to time. Here precious time of minute has calculated by comparing values of loop and real time clocks playing essential part in removing additional external hardware.

2.1.8Delay for stability

Figure 9 shows the delay for visually stable view.

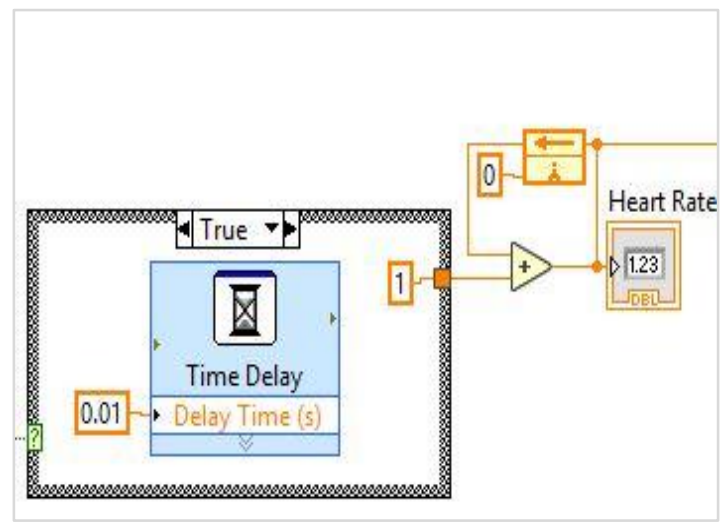

Figure 9 Delay for visually stable view
Delay unit of 0.01 second has cascaded along with to enhance stability of the received signal. Arrow of feedback represent increment of heart rate by single number every time R-R peak interval occurs, so counting up heart rate for every minute and resetting at the inception of another minute after giving notification of result of cardiac condition whether it is normal or suffering with tachycardia and bradycardia.

\subsubsection{Heart rate calculation}

Heart rate calculation has achieved here using R-R interval of the ECG waveform pertaining to comparatively equal with entire normal ECG waveform duration of about 0.8 seconds. To 1.2 seconds. Therefore if R-R interval count got inclines or declines than particular limit it can be concluded as arrhythmia which got assigned further by its mathematical calculation.

\section{Flow chart}

A detailed description has provided here to narrate the working procedure of this innovative and less expensive technique. Flowchart illustrates here from beginning of preparation to consequence achieved.

1. Patient/User need to have manual electrode placement at particular places for conduct electrocardiography test.

2. ECG Acquisition circuit to acquire minimally signal occurred due to electrical movement of the heart thankful to atriovetricular node.

3. To provide extra safety and eradicate chances of electrical hazards might be happen as a result of current leak or reverse current during test with lack of expert observation

4. This Technique works based on simultaneous use of controller and LabView platform for provide customized platform and avoid extra expense of patient for global prevalence.

5. VISA (Virtual instrumentation software architecture) installation is mandatory to commute LabView serially with controller installed in operating system. So inception of LabView will make determination of whether drivers are available or not and will notify with prompt notification and recommendation.

6. Scrutiny need to be conduct every time VI get started by user to determine electrode placement is proper to ensure outcomes is appropriate to consider for further guidance of physician with data validation. Which reveals certain repetitive data in case electrodes are attached at the surface according to recommendation. 
7. Entire algorithms start reading data for entire single minute to extracts and recognise QRS segment event to statistical counting.

8. Computed result get compared with sort of data already given to controller to ensure the type and severity of the arrhythmia and display on LabView.

Figure 10 shows the flowchart of the proposed method.

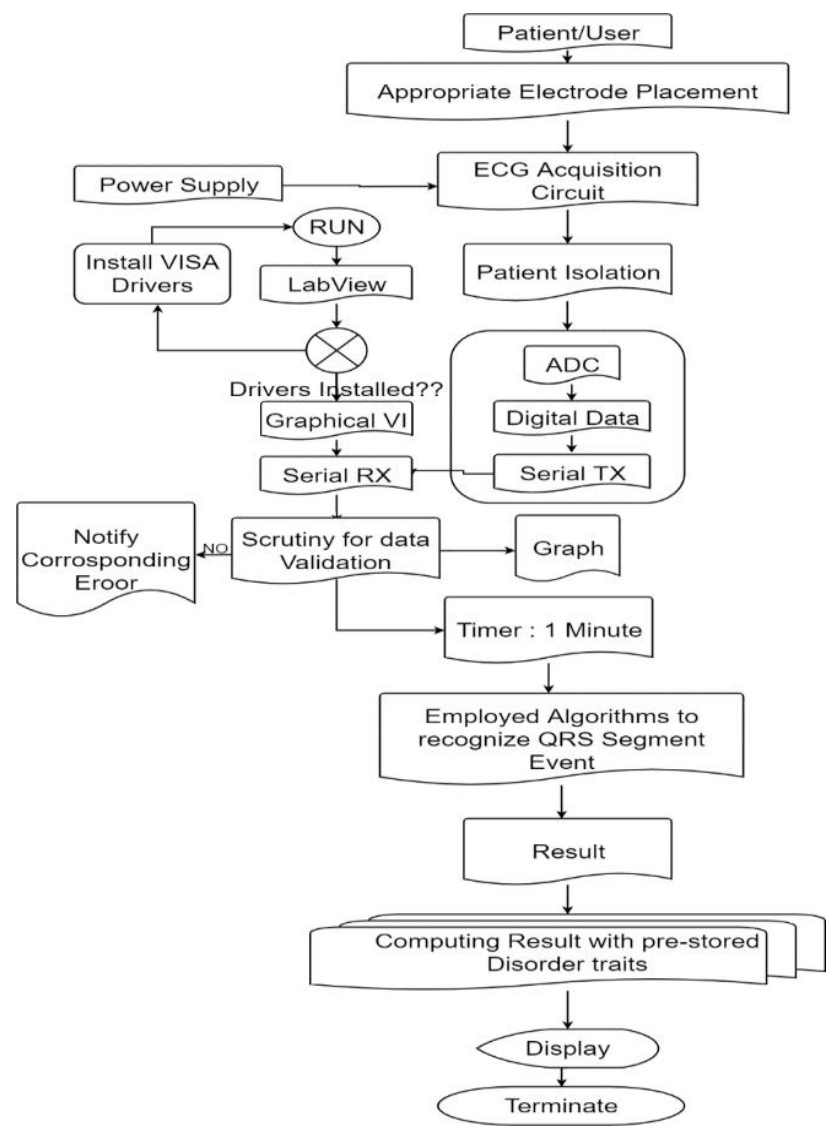

Figure 10 Flowchart of proposed method

\section{Result}

LABVIEW GUI has been illustrated below with simple interpretation by determination of heart rate using R-R interval since Healthy user seems to have waveform are more natural and rhythmic not suffering with any fibrillation of heart. ECG of normal user has depicted here in figure revealing range lies under normal ECG waveform (Figure 11).

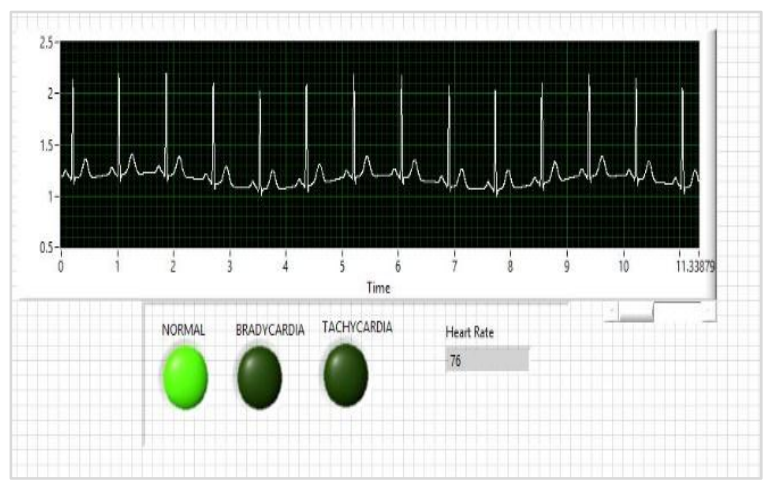

Figure 11 Normal ECG consequences in LabView GUI

This depicted screenshot below reveals the ECG output of patient which can be considered for bradycardia, since there are more distance between two waves and less number of R-R interval in entire block of 12seconds (Figure 12). It has showing heart rate of 45 .

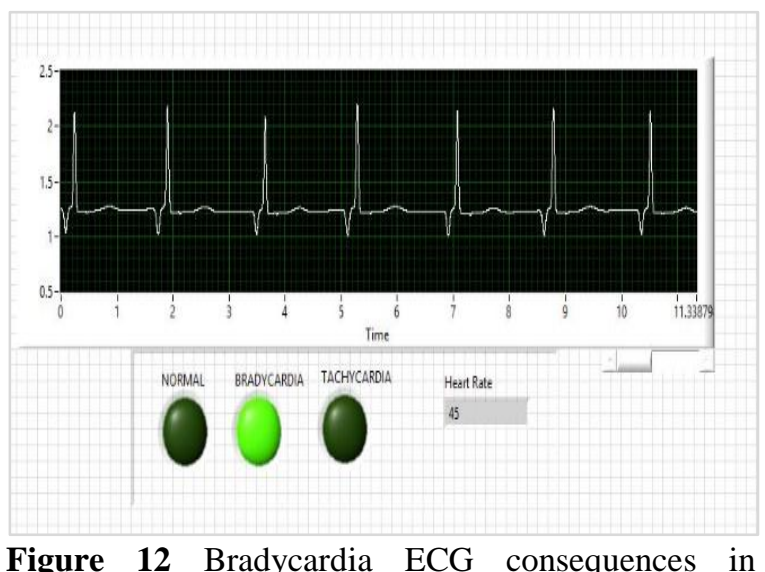

LabView GUI

Below image represents rapid ECG waves has mapped in entire duration of 12 seconds so all three waves can be discriminated from each other. Tachycardia has been determined with this incident and highlighted with indication LED since this was achieved on healthy patient by behest him to run as fast as possible with to acquire this waveforms (Figure 13). 


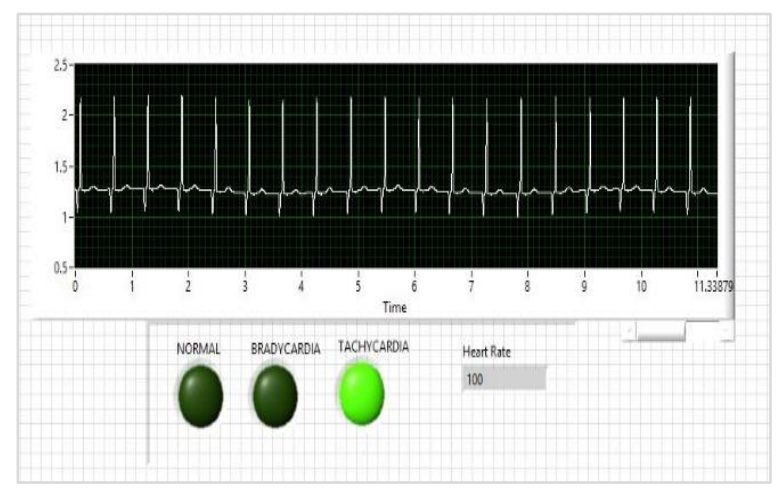

Figure 13 Tachycardia ECG consequences in LabView GUI

\section{Discussion}

Inspiration was to detect arrhythmia at early stage at affordable cost for everyone. Which can be portable and easily accessible, there are some rural places where hospital is not nearby. So they need to travel long every day to take assistant of physician. But using this platform ECG data can be stored and analyse as well provide accessibility to know result with graphical indication. So it can be forward to expert whenever required which has not become possible and convenient for everyone in early times. Since traditional ECG technique makes use of same electrode we had used but a complex circuitry with limited functionality of printing graph on paper only which requires a detailed study and lot of hand on experience to understand. In Contrast aforementioned method gives accessibility to check whether electrode are placed properly or not with facility of automatically identification of relevant arrhythmia at the end of examination saves time and promotes to take further guidance from the experts. Challenging task in this experiment was acquiring EMG signal and compact the size of circuit as possible. As we had undergone through depth research and found circuitry which had use instrumentation amplifier as well low pass and band pass filter separately which takes relatively more place than assembled version of IC we had use. We had utilized this experiment with portable battery of $5 \mathrm{~V}$ whereas $9 \mathrm{~V}$ is recommended by manufacturer to get smoother signal. We had tried omitting $9 \mathrm{v}$ and use $5 \mathrm{v}$, which has given satisfactory result to determine ECG waveform required for arrhythmia detection. Reason behind use this with DC portable supply was to eliminate need of notch filter which is required and must in case of using $\mathrm{AC}$ supply since it gives fluctuate frequency at particular period. Rather than using Biomedical Toolkit or express VI to acquire ECG signal we had tried working with serial interfacing with baud rate 9600 to design VI which can be modified according to our requirement and does not involves complex steps to debug any error encountered during acquisition and execution.

\section{Conclusion and future work}

Pre-estimating cardiac arrhythmia using LabView based GUI approach is novel way to recognize heart condition of the victim or any user virtually. Since it is need so much expensive and recommend heavy expense during every time of heart condition determination this technique can be employed for individual and personal purposes. Main objective behind forcing graphical configuration is to adding up more and more features with the same hardware which doesn't restrict progress at some horizons or boundary. Status of sinus bradycardia, sinus tachycardia and fibrillation of SA node has determined with this technique. Adding up new feature of predicting rather abnormality of heart involves summation of theoretical calculation only with appropriate electrode placement.

Future work involves cloud server and android application based monitoring which resemble to give notification about alerting changes regards to victim and status of victim. So this can be connected with physician so immediate steps can be taken by physician or surgeon and ambulatory services can be provided.

\section{Acknowledgment}

None.

\section{Conflicts of interest}

The authors have no conflicts of interest to declare.

\section{References}

[1] Rege S, Barkey T, Lowenstern M. Heart arrhythmia detection. Virtual conference on applications of commercial sensors (VCACS) 2015 (pp. 1-7). IEEE.

[2] Curtis AB, Karki R, Hattoum A, Sharma UC. Arrhythmias in patients $\geq 80$ years of age: pathophysiology, management, and outcomes. Journal of the American College of Cardiology. 2018; 71(18):2041-57.

[3] Al-Fahoum AS, Qasaimeh AM. ECG arrhythmia classification using simple reconstructed phase space approach. In computers in cardiology 2006 (pp. 75760). IEEE.

[4] Acharya R, Kannathal N, Krishnan SM. Comprehensive analysis of cardiac health using heart rate signals. Physiological Measurement. 2004; 25(5):1139-51.

[5] Rathikarani V, Dhanalakshmi P. Automatic classification of ECG signal for identifying 
arrhythmia. International Journal of Advanced Research in Computer Science and Software Engineering. 2013; 3(9): 10376-84.

[6] Cromwell L, Weibell FJ, Pfeiffer EA. Biomedical instrumentation and measurements. Pearson; 2018.

[7] Murugappan M, Thirumani R, Omar MI, Murugappan S. Development of cost effective ECG data acquisition system for clinical applications using LabVIEW. In international colloquium on signal processing \& its applications 2014 (pp. 100-5). IEEE.

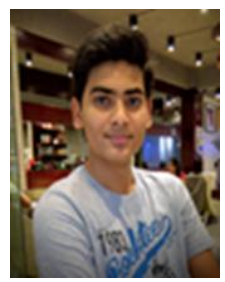

Mr. Abhijeet R. Satani researching on computer brain interface and also written a book "A Beginners Guide For Ethical Hacking". He is first to invent Wirelese Computer Brain Interface in 2014 and successfully grant a patent. $\mathrm{He}$ is the founder of Limbable Biotechnology Private Limited.

Email: abhijeetsatani@gmail.com

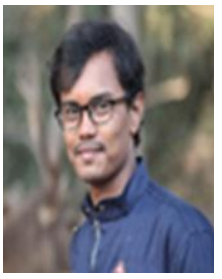

Mr. Dinesh R. Damodar currently pursuing his skills in research as a independent researcher and selfemployed. $\mathrm{He}$ has pursued his bachelors in Biomedical engineering from Gujarat Technological University particularly from Government Engineering College. $\mathrm{He}$ is aspiring towards healthcare industry innovation. Major area of interest are Rehabilitaion Instrumentaton, Neuroscience, Biomechanis, Biomedical Instrumenation, Artificial Intelligence, Robotics, Machine learning.

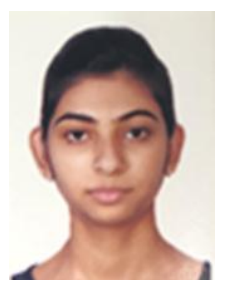

Dr. Bhoomi satani currently completed her MBBS from SMT NHL Municipal Medical College. She is director of Limbable Biotechnology Private Limited. 